\title{
Active mid IR plasmonics using giant magneto resistance
}

Gaspar Armelles, Alfonso Cebollada, Fernando García, Lorena Torné, María Luisa Dotor

Gaspar Armelles, Alfonso Cebollada, Fernando García, Lorena Torné, María Luisa Dotor, "Active mid IR plasmonics using giant magneto resistance," Proc. SPIE 10357, Spintronics X, 103571Q (6 September 2017); doi: $10.1117 / 12.2273690$

Event: SPIE Nanoscience + Engineering, 2017, San Diego, California, United States 


\title{
Active Mid IR plasmonics using Giant Magneto Resistance Gaspar Armelles, Alfonso Cebollada, Fernando García, Lorena Torné and María Luisa Dotor IMM-Instituto de Microelectrónica de Madrid (CNM-CSIC) Isaac Newton 8, PTM, E-28760 Tres Cantos, Madrid, Spain
}

\begin{abstract}
Here we present our proposal and initial results on the magnetic field control of plasmon resonances in the mid IR region by the use of the Magneto-Refractive (MR) effect, i.e., a change in the optical properties of the system by magnetic field controlled electrical resistivity. For this we select a Giant Magneto Resistance model system (a Au/Permalloy multilayer), for which changes in resisitivity of the order of $10 \%$ by the application of small (of the order of $20 \mathrm{Oe}$ ) magnetic fields have been reported. The experiments are carried out in a dedicated magnetic field FTIR (M-FTIR) spectrometer.
\end{abstract}

Keywords: Plasmonics, Mid-IR, Giant Magnetoresistance, Magnetorefractive.

\section{INTRODUCTION}

Plasmonics has become a powerful tool to improve the performance of mid-IR devices, resulting in plasmon assisted quantum cascade lasers, plasmon enhanced light detection, plasmonic beam steering, plasmonic thermal emitters or plasmonic nanoantenas for vibrational spectroscopy. In this context, the possibility of modulating the emission, propagation and/or detection of mid-IR radiation by acting on the plasmon properties constitutes a promising aspect to expand the limits of the currently used technologies. In this sense, fast and contactless actuation on plasmon resonances via the Magneto-Optical (MO) effect has been achieved by the inclusion of ferromagnetic components into noble metal layers and nanostructures (magnetoplasmonic nanostructures), but restricted to the visible and near-infrared ranges. In these systems plasmon properties can be controlled by applying an external magnetic field due to the magneto optical MO activity of the ferromagnet, directly related with the non-diagonal elements of its dielectric tensor. The Magneto Optic Effect (MOE) has a linear dependence with the magnetization, and has been used to modulate the wave vector of propagative surface plasmon modes ${ }^{1}$ due to the magnetization reversal effect on the off diagonal elements on the dielectric tensor (Fig. 1-left). This property has been applied to development sensors and MO active devices ${ }^{2,3,4}$, improving the performance of currently available non-MO counterparts. Unfortunately the MOE effect weakens beyond the near IR, making this effect not suitable for the implementation of active devices in the mid-IR and longer wavelengths ranges. However, a magnetic field can act, not only on the non-diagonal elements of the dielectric tensor of a ferromagnetic system, but also on the diagonal ones ${ }^{5}$. In particular, it has been shown that in some ferromagentic/non ferromagnetic multilayers a magnetic field modifies the diagonal elements of the effective dielectric tensor of the multilayer. This magnetic field induced diagonal effect, known as magnetorefractive effect (MRE) ${ }^{6}$, is quadratic with the magnetization (Fig 1-right), and interestingly reaches sizable values in the mid-infrared and $\mathrm{THz}$ regimes ${ }^{7,8}$. Its origin is related to the strong magnetic field induced changes of the conductivity, the so called Giant Magneto Resistance $(\mathrm{GMR})^{9,10}$, due to the different electrical conductivities characteristic of a parallel or antiparallel configuration of the individual ferromagnetic layer's magnetizations in magnetic multilayers. In these spectral ranges, the optical response of the metallic multilayers is determined by the conduction electrons and, therefore, the changes in the dc conductivity are connected with appreciable changes of the diagonal elements of the effective dielectric tensor. Therefore, the MRE can be an alternative to the MOE to magnetically modulate plasmon properties in the mid IR range.

In this work, we present the first experimental evidence of magnetic field modulation of plasmon properties in the midIR range using GMR structures. Among the different systems that present GMR properties we have selected the $\mathrm{Ni}_{81} \mathrm{Fe}_{19} / \mathrm{Au}$ multilayer due to its reported well-balanced characteristics in all the factors of relevance: relative large GMR values, of up to $12 \%$, along with saturation magnetic fields as low as $50 \mathrm{Oe}^{11}$. In addition, $\mathrm{Au}$ is an excellent plasmonic material from the optical range up to wavelengths of around some microns.

Spintronics X, edited by Henri-Jean Drouhin, Jean-Eric Wegrowe, Manijeh Razeghi, Henri Jaffrès, Proc. of SPIE Vol. 10357, 103571Q · @ 2017 SPIE · CCC code: 0277-786X/17/\$18 · doi: 10.1117/12.2273690 


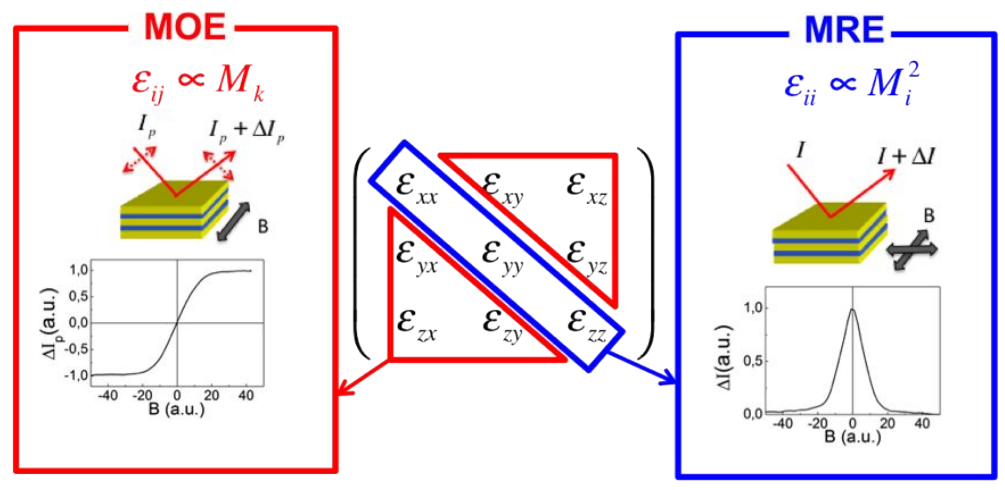

Fig. 1. Magneto Optic (left) and Magneto Refractive (right) effects (MOE and MRE) are related to the non-diagonal and the diagonal elements of the dielectric tensor (center), respectively.

\section{MATERIALS AND RESULTS}

\subsection{GMR multilayer system.}

The layers were grown on $\mathrm{CaF}_{2}(111)$ single crystalline substrates, which offer an excellent platform for high quality sharp interfaces growth of the multilayers. After transfer into the ultrahigh vacuum deposition a $2 \mathrm{~nm}$ Ti buffer layer was grown by electron beam evaporation. Then, the $\mathrm{Ni}_{81} \mathrm{Fe}_{19} / \mathrm{Au}$ multilayers were deposited by magnetron sputtering from individual $\mathrm{Ni}_{81} \mathrm{Fe}_{19}$ and $\mathrm{Au}$ targets at an Ar pressure of $10^{-3}$ mbar. The substrate temperature was kept at $150^{\circ} \mathrm{C}$ during the whole process. In this work, a series of samples were grown, being the thickness of the $\mathrm{Ni}_{81} \mathrm{Fe}_{19}$ layers fixed, to a value of $2.9 \mathrm{~nm}$, and the thickness of the Au layers varied around $2.4 \mathrm{~nm}$. A small change in the Au thickness gives rise to multilayers with very similar optical and magnetic properties, but with different GMR values ${ }^{11}$. The total thickness of the multilayers varies between $66 \mathrm{~nm}$ to $72 \mathrm{~nm}$ around the series.

\subsection{Experimental results}

a)

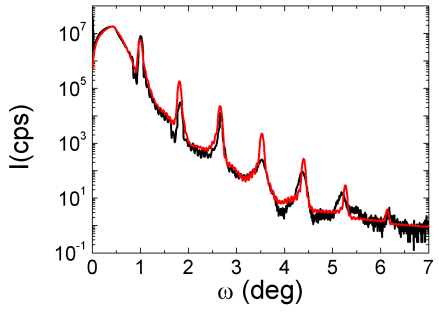

b)

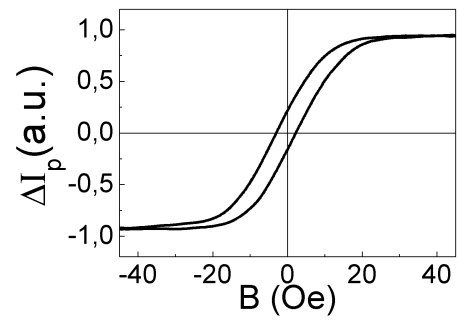

c)

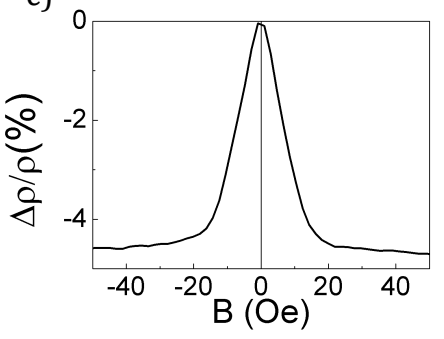

Fig. 2. a) XRR measurements on selected multilayer (black) and corresponding fit (red). b) TMOKE hysteresis loop for the same multilayer. c) Magnetorresistance curve for a 4,8\% GMR multilayer.

In figure 2 (a) we present X-ray reflectometry measurements of one of the grown samples. The black curve corresponds to the experimental measurement, whereas the red curve represents a fit using a standard code. Such procedure allows a precise determination of the individual layers thickness and interface roughness. Prior to the optical characterization measurements, Transverse MOKE (TMOKE) hysteresis loops were performed to characterize the magnetic behaviour of the structures, whereas the electrical properties were analysed by DC Magneto resistance measurements using 4 probes in line. In figure 2 (b) and figure 2 (c) we present TMOKE hysteresis loops and DC magneto resistance curves of a 
characteristic sample. The TMOKE loops were obtained at an incident angle of 50 degrees using a green laser source. As it can be observed, the magnetic field needed to saturate the samples can be as low as a few Oe, and the GMR value obtained is around $4.8 \%$. Probably the somehow lower interface quality in our sputtered multilayers with respect to the MBE grown in [11] can justify not reaching the previously reported $12 \%$ GMR values. The IR optical and magnetooptical properties of the structures where obtained using a Bruker VERTEX 70 FTIR spectrometer equipped with a MCT photovoltaic detector and magnetic coils in the sample's enclosure, generating sufficient magnetic field to saturate the structures. A sketch of the configuration measurement is shown in figure 3 (a).
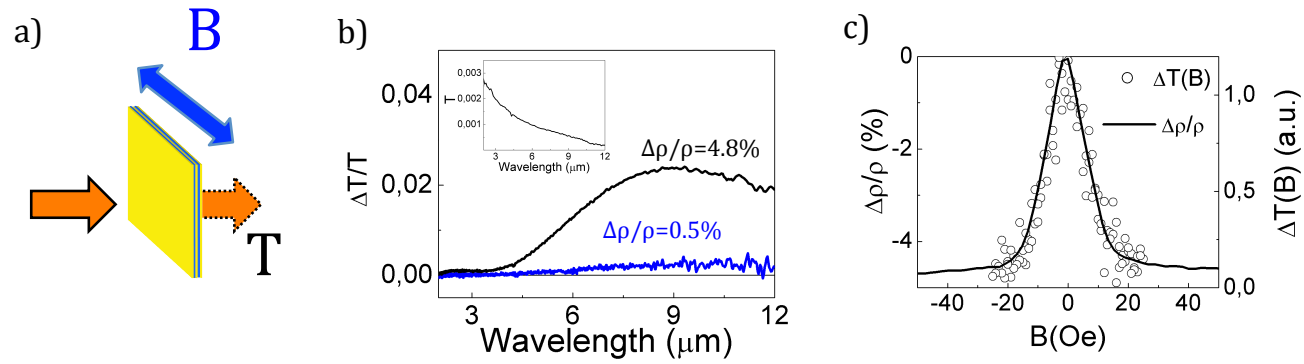

Fig. 3. a) Sketch of the M-FTIR measurement configuration. b) Magnetic field induced relative change of transmission for a multilayer showing 4,8\% GMR (black) and for a sample with 0,5\% GMR (blue). Inset, shows the transmission at normal incidence for a standard multilayer. c) Magnetic field dependence of the resistivity change (continuous line) and of the change of IR transmission (circles). The perfect overlap confirms the direct relation between both physical effects.

In the inset of figure 3 (b) we present a transmission spectrum at normal incidence for unpolarized light of a representative multilayer. The spectrum shows a strong absorption of the metallic layer with a decrease of the transmission as we increase the wavelength. On the other hand, in Figure 3 (b), we also show the relative change in the transmission $\left(\Delta T / T, \Delta T=T\left(M_{\text {sat }}\right)-T(0)\right.$ at normal incidence induced by an applied magnetic field for two different $\mathrm{Ni}_{81} \mathrm{Fe}_{19} / \mathrm{Au}$ multilayers with different GMR values( $4.8 \%$ and $\left.0.5 \%\right)$. The magnetic field was applied in plane and its intensity was sufficient to saturate the samples. The incident light was not polarized. As it can be observed, the value of $\Delta T / T$ increases as we increase the wavelength and shows a maximum at around $9 \mu \mathrm{m}$, slightly decreasing for larger wavelengths. Besides, the magnitude of $\Delta T / T$ is larger for the multilayer which has a higher value of GMR, indicating the relation between both effects. This is better seen in figure $3(\mathrm{c})$, where we present the magnetic field dependence of $\Delta T(B)\left(\Delta T(B)=T\left(M_{\mathrm{sat}}\right)-T(\mathrm{~B})\right)$ and the GMR curve. As it can be observed, both magnitudes show the same magnetic field dependence, quadratic with the magnetization, corroborating the relationship between GMR and $\Delta T / T$, being the magnetic field induced changes of the transition due to MRE.

a)

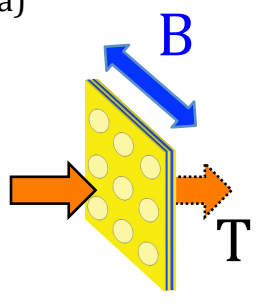

b)

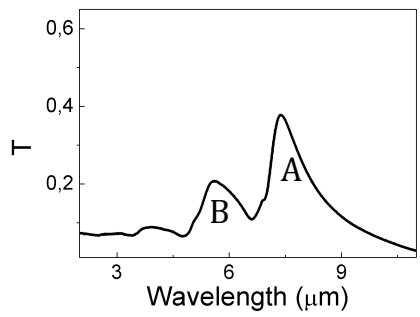

c)

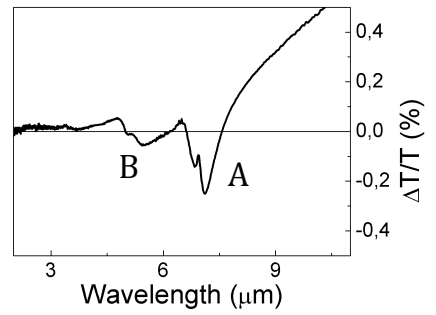

Fig. 4. a) Sketch of measurement configuration. b) Normal incidence transmission spectrum for a perforated multilayer, showing the characteristic EOT peaks that indicate the excitation of plasmon resonances. c) Magnetic field induced relative change for a perforated multilayer exhibiting 4,8\% GMR. The clear derivative lineshape with respect to the transmission spectrum indicates the clear modulation character induced by the magnetic field via the MRE effect. 
Finally, in order to analyse the utility of MRE to modulate plasmon properties in the mid-IR range we have fabricated ordered arrays of holes in an Extraordinary Optical Transmission (EOT) ${ }^{12}$ fashion, on multilayers with and without GMR, using photolithography and ion etching. We have checked that this process does not change the magnetic and electric behaviour of the multilayer, obtaining similar (not shown) TMOKE hysteresis loops and unaltered GMR values. The period of the array was $5,1 \mu \mathrm{m}$ and the diameter of the holes $2.5 \mu \mathrm{m}$. These membranes allow the coupling of photons to plasmons, giving rise to extraordinary transmission (EOT) effects. In figure 4 (b) we show the transmission spectrum at normal incidence of one of a membrane with $4.8 \%$ GMR for unpolarized light. In the spectrum we observe two main features (peaks) related to the excitation of plasmons at the metal $\mathrm{CaF}_{2}$ interface (A-region) or at the metal air interface (B-region). On the other hand in figure 4 (c) we present the normalized magnetic field induced change in the transmission, shown in figure 4 (b), with clear derivative-like features in the spectral region of the EOT peaks, and clearly suggesting magnetic modulation of plasmon properties induced by MRE. Besides, measurements on structures fabricated using multilayers of similar composition but with no GMR effect produced no magnetic plasmon modulation, confirming the role of the MRE as the physical mechanism for this modulation

In conclusion, by using ordered arrays of membranes fabricated on $\mathrm{Ni}_{81} \mathrm{Fe}_{19} / \mathrm{Au}$ multilayers with GMR, we have shown that magnetic modulation of plasmon properties can be achieved via the MRE effect, opening the door to a new approach to fabricate active structures in the mid-IR or THz range.

We acknowledge financial support from MINECO through project AMES (MAT 2014-58860-P). We also acknowledge the service from MiNa Laboratory at IMM, and funding from CM under project S2013/ICE-2822 (Space-Tec) with support from EU (FEDER, FSE).

\section{REFERENCES}

[1] González-Díaz, J.B., García-Martín, A., Armelles, G., García-Martín, J.M., Clavero, C., Cebollada, A., Lukaszew, R.A., Skuza, J.R., Kumah, D., and Clarke, R., "Surface magnetoplasmon non-reciprocity effects in noble metal/ferromagnetic heterostructures," Phys. Rev. B, 76, 153402 (2007).

[2] Sepúlveda, B., Calle, A., Lechuga, L.M., and Armelles, G., "Highly sensitive detection of biomolecules with the magneto-optic surface-plasmon-resonance sensor," Opt. Lett. 31, 1085-1087 (2006).

[3] Sepúlveda, B., Lechuga, L.M., Armelles, G., "Magnetooptic effects in surface-plasmon-polaritons slab waveguides," Journal of Lightwave Technol. 24, 945-955 (2006).

[4] Temnov, V.V., Armelles, G., Woggon, U., Guzatov, D., Cebollada, A., García-Martín, A., García-Martín, J.M., Thomay, T., Leitenstorfer, A., and Bratschitsch, R., "Active magneto-plasmonics in hybrid metal-ferromagnet structures," Nat. Phot. 4, 107-111 (2010).

[5] Sepúlveda, B., Huttel, Y., Martínez-Boubeta, C., Cebollada, A., and Armelles, G., "Linear and quadratic magnetooptical Kerr effects in continuous and granular ultrathin monocrystalline Fe films," Phys. Rev. B 68, 064401 (2003).

[6] Jacquet, J.C., and Valet, T.A., "A new magnetooptical effect discovered on magnetic multilayers: the magnetorefractive effect,” Mat. Res. Soc. Symp. Proc. 384, 477-490 (1995).

[7] Vopsaroiu, M., Stanton, T., Thomas, O., Cain, M., and Thompson, S.M., "Infrared metrology for spintronic materials and devices," Meas. Sci. Technol. 20, 045109 (2009). 
[8] Jin, Z., Tkach, A., Casper, F., Spetter, V., Grimm, H., Thomas, A., Kampfrath, T., Bonn, M., Kläui, M., and Turchinovich, D., "Accessing the fundamentals of magnetotransport in metals with terahertz probes," Nat. Phys. 11, 761-766 (2015).

[9] Baibich, M.N., Broto, J.M., Fert, A., Nguyen Van Dau, F., Petroff, F., Etienne, P., Creuzet, G., Friederich, A., and Chazelas, J., "Giant magnetoresistance of (001)Fe/(001)Cr magnetic superlattices," Phys. Rev. Lett. 61, 2472-2475 (1988).

[10] Binasch, G., Grünberg, P., Saurenbach, F., and Zinn, W., "Enhanced magnetoresistance in layered magnetic structures with antiferromagnetic interlayer exchange," Phys. Rev. B 39, 4828-4830 (1989).

[11] Parkin, S.S.P., Farrow, R.F.C., Marks, R.F., Cebollada, A., Harp, G.R., and Savoy, R.J., "Oscillations of interlayer exchange coupling and giant magnetoresistance in (111) oriented permalloy/Au multilayers," Phys. Rev. Lett. 72, 37183721 (1994).

[12] Ebessen, T.W., et al., Nature 391, 667 (1998). 